\title{
Phase field modeling of convective and morphological instability during directional solidification of an alloy
}

\author{
C.W. Lan*, M.H. Lee, M.H. Chuang, C.J. Shih \\ Department of Chemical Engineering, National Taiwan University, Taipei 10617, Taiwan, ROC
}

Received 16 March 2006; received in revised form 22 July 2006; accepted 26 July 2006

Communicated by G.B. McFadden

\begin{abstract}
Phase field modeling is carried out to investigate the convective and morphological instability during directional solidification of a succinonitrile/acetone alloy. Considering the presence of gravity, we have found that the planar interface could become wrinkled even beyond the Mullins-Sekerka instability; this is originated from the lateral solute segregation induced by the flow. For the cases slightly above the onset of instability, morphologies of shallow cells are affected by the convection as well. The cells with different wavelengths and depths can coexist due to the flow-induced segregation. The coupling of long- (convective mode) and short wavelengths (morphological mode) is illustrated for the first time, which cannot be predicted by the linear stability theory. As the growth rate is further increased, the effect of the buoyancy decreases.
\end{abstract}

(C) 2006 Elsevier B.V. All rights reserved.

PACS: 68.70. +w; 81.30.Fb

Keywords: A1. Adaptive phase field simulation; A1. Convection; A1. Morphological instability; A1. Solidification

\section{Introduction}

During alloy directional solidification, as the constitutional supercooling occurs to a certain degree, cellular or dendritic patterns develop as a result of the interplay of solute, temperature, and flow fields with the solidification interface. Without convection, the planar interface becomes unstable at a certain critical value of the control parameter, which is either the pulling speed $(V)$ or the temperature gradient $(G)$, as described by the classical Mullins-Sekerka (MS) theory [1]. The morphological instability may interact with the convection, and this was firstly realized by Coriell et al. [2] by using a linear stability analysis in the presence of thermosolutal convection in the melt. Two modes of instability were identified, which were distinguishable by the spatial wavelength of the interface. The morphological mode was found consistent with the prediction of the MS theory, while the long-wavelength

\footnotetext{
${ }^{*}$ Corresponding author. Tel./fax: + 886223633917.

E-mail address: cwlan@ntu.edu.tw (C.W. Lan).
}

branch, i.e., the convective mode, was not affected by the interface. In between, some interaction region leading to an oscillatory state was found. Coriell and McFadden [3] further performed linear stability analyses for a tin containing lead alloy, for both stabilized thermal and solutal fields. They also showed that the minima of the neutral stability curves occurred at much longer wavelengths, sometimes on the order of ampoule diameter, than the minima of the purely morphological stability. In an interesting directional solidification experiment by Schaefer and Coriell [4] using succinonitrile containing ethanol in an ampoule, it was illustrated that the interface deformation, especially the pit formation at the center of the interface, was caused by thermal convection. The pit formation was further revealed through computer simulation by Lan and $\mathrm{Tu}$ [5], and the onset of the morphological instability was significantly earlier than that predicted by MS theory. Fully nonlinear analyses by using a finite element method with symmetry boundaries were also investigated by Mehrabi in Brown's group [6]. Given the width (half of the wavelength) of the computational domain, the 
interaction of the flow and the morphological evolution was illustrated for the convective mode. Unfortunately, their simulation was not able to simulate the appearance of the both modes due to the limited mesh resolution and deformation. In fact, to cover a large domain accommodating the long (convective mode) and short (morphology mode) wavelengths and to allow complex morphological evolutions for a solidification simulation is not trivial, especially for the front-tracking approach.

The phase field model has emerged as a powerful tool for simulating solidification problems with a complex morphological evolution (e.g., Refs. [7-9]). However, quantitative simulation of alloy solidification is not trivial. An extremely small interface thickness (on the order of $0.1 \mu \mathrm{m}$ ) is required for thermodynamic consistency, while a large domain $(>1000 \mu \mathrm{m})$ is needed to accommodate the solute boundary layer and characteristic wavelengths. In addition, solute trapping [10] can be induced due to the diffuse interface, especially at high solidification speed. Fortunately, this problem was first resolved by Karma [11] using the anti-trapping model and by Shih et al. [12] using a simple interface model. Furthermore, with the use of the adaptive grid [13], a quantitative simulation for alloy solidification has become feasible.

In this report, the adaptive phase field method [13] using the Karma's anti-trapping model is adopted to simulate the directional solidification of a succinonitrile $(\mathrm{SCN}) /$ acetone alloy under an unstable solutal field near the onset of the MS instability. The interaction of the convection and the cell development is illustrated for the first time; in fact, they are indistinguishable as pointed out by Mehrabi [6]. In the next section, the phase field model used in the simulation is briefly described. Section 3 is devoted for results and discussion, followed by a short conclusion.

\section{Phase field model}

The computational domain investigated in this study is sketched in Fig. 1, and a two-dimensional case is adopted for simplicity. The thermal profile is assumed to be linear having a gradient $G$ as a working approximation. As the solidification starts, the light solute (acetone) is rejected from the solid and accumulates near the melt/solid interface to reduce the density there. As discussed in Ref. [2], for thermosolutal convection the stability criterion is more complicated than simply examining the sign of the net density gradient. The mechanism is instead based on the different rates of diffusion of heat and solute ("doublediffusion").

Therefore, in the presence of gravity, this destabilizes the system, as illustrated in Fig. 2c. Away from the interface, the system is stabilized by the thermal effect; Figs. $2 \mathrm{a}$ and $\mathrm{b}$ describe schematically the corresponding destabilizing concentration and stabilizing thermal profiles, respectively. Therefore, to a certain degree of acetone accumulation, convection is induced due to hydrodynamic instability. The convection leads to lateral segregation and interacts with

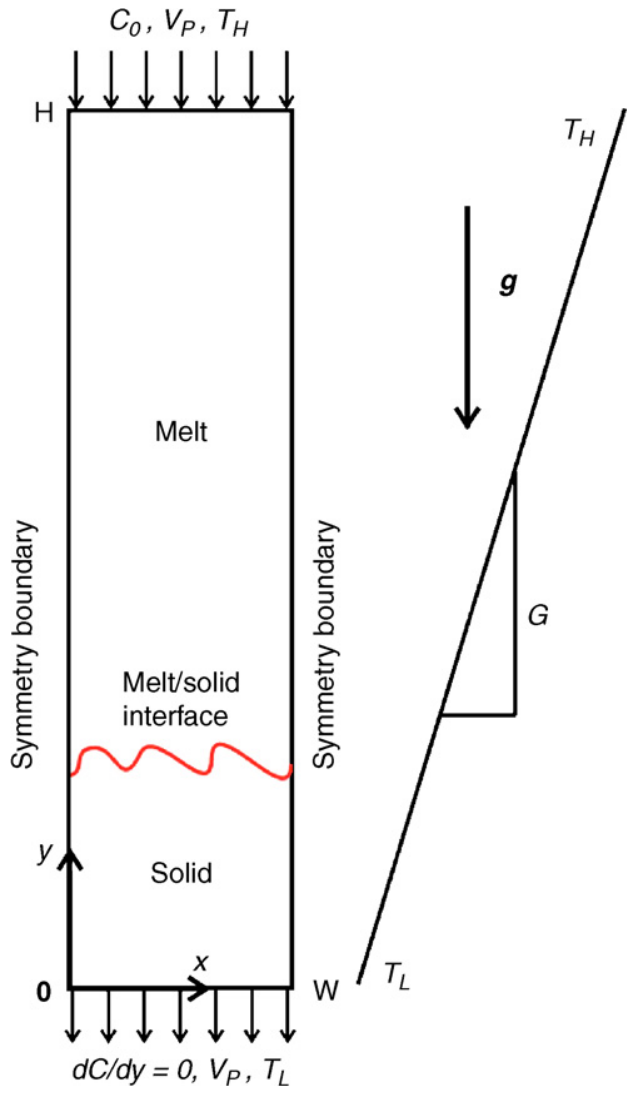

Fig. 1. Schematic of the computational domain for directional solidification under gravity.

the development of the local interface morphology. In order to simulate the physical problem, an adaptive phase field model is used.

Similar to the volume-averaged derivation by Beckermann et al. [14], the two-dimensional phase field equation for an alloy can be obtained as the following:

$$
\begin{aligned}
\eta^{2} \frac{\partial \phi}{\partial t}= & \Gamma \mu_{\mathrm{A}}\left[\nabla \cdot\left(\eta^{2} \nabla \phi\right)-\frac{\partial}{\partial x}\left(\eta \eta^{\prime} \frac{\partial \phi}{\partial y}\right)\right. \\
& \left.+\frac{\partial}{\partial y}\left(\eta \eta^{\prime} \frac{\partial \phi}{\partial x}\right)-\frac{\phi(1-\phi)(1-2 \phi)}{\delta^{2}}\right] \\
& -\mu_{\mathrm{A}}\left(T_{m}^{\mathrm{A}}-T-|m| c_{\mathrm{L}}\right)\left[\frac{5 \phi^{2}(1-\phi)^{2}}{\delta}\right],
\end{aligned}
$$

where the anisotropic function $\eta$ is defined for the four-fold symmetry, i.e., $\eta=1+\varepsilon \cos 4 \Theta$ while $\varepsilon$ is the intensity of the anisotropy and $\Theta=\tan ^{-1}[(\partial \phi / \partial y) /(\partial \phi / \partial x)]$ is the angle between the direction normal of the interface and the $x$ (horizontal) axis. The phase field variable $\phi(x, y, t)$ changes rapidly from 0 (solid) to 1 (liquid) at the interface. $\Gamma$ is the Gibbs-Thomson coefficient, $\mu_{\mathrm{A}}$ the kinetic coefficient, and $m$ the slope of liquidus temperature line $\left(T_{i}\right) ; T_{m}^{\mathrm{A}}$ is the melting point of pure $\mathrm{A}(\mathrm{SCN})$ and $c_{\mathrm{L}}$ the concentration of the solute (acetone) at the liquid phase side. Furthermore, $\delta$ is the thickness of the diffuse interface. The corresponding energy (for temperature $T$ ) and species (for the acetone concentration $C$ ) equations are 

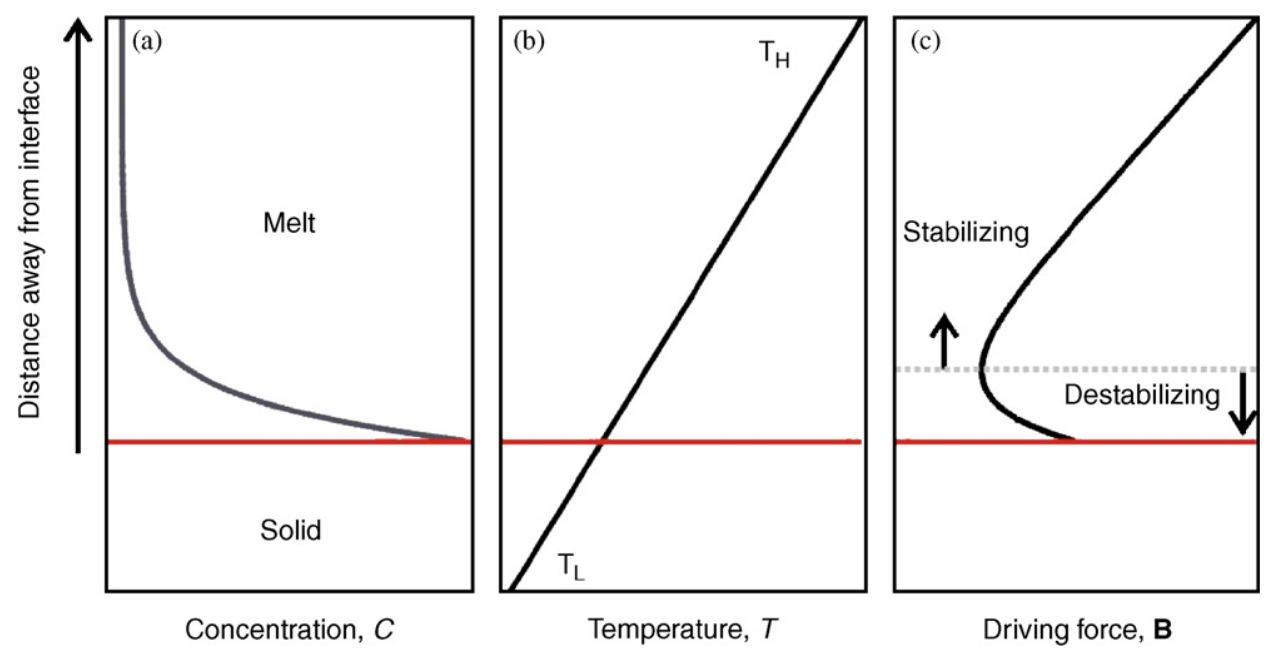

Fig. 2. Schematic illustration of the axial distribution of (a) concentration, (b) temperature, and (c) buoyancy driving forces causing the convective instability during directional solidification; the destabilizing region exists only near the interface.

also obtained as follows:

$\frac{\partial T}{\partial t}+\nabla \cdot(p(\phi) \boldsymbol{V} T)=\alpha \nabla^{2} T-\frac{L_{A}}{C_{p}} \frac{\partial \phi}{\partial t}$,

$\frac{\partial C}{\partial t}+\nabla \cdot\left(\frac{p(\phi) C}{p(\phi)+k(1-p(\phi))} \boldsymbol{V}\right)$

$=\nabla \cdot \tilde{D}\left[\nabla C-\frac{(1-k) p^{\prime}(\phi) C}{p(\phi)+k[1-p(\phi)]} \nabla \phi+\frac{1}{\tilde{D}} \boldsymbol{j}_{\mathrm{atc}}\right]$,

where

$\tilde{D}=D_{\mathrm{S}}+\frac{\left(D_{\mathrm{L}}-D_{\mathrm{S}}\right) p(\phi)}{p(\phi)+k(1-p(\phi))}$.

Also,

$\boldsymbol{j}_{\text {atc }}=\delta(1-k)\left[\frac{C}{p(\phi)+k(1-p(\phi))}\right] \frac{\partial \phi}{\partial t} \frac{\nabla \phi}{|\nabla \phi|}$

is the anti-trapping current proposed by Karma [11] to suppress the effect of solute-trapping. $D_{\mathrm{S}}$ and $D_{\mathrm{L}}$ are the solute diffusivities in the solid and liquid phases, respectively and $p(\phi)$ is an interpolation function to describe solid/melt mixture properties in the diffuse interface; $p(\phi)=\phi$ is used here. In addition, $k$ is the equilibrium segregation coefficient, $\alpha$ the thermal diffusivity, $L_{\mathrm{A}}$ the latent heat, and $C_{p}$ the specific heat. The continuity and momentum equations for the pressure $P$ and the velocity vector $\boldsymbol{V}$ can further be derived as:

$\nabla \cdot\left(p(\phi) \rho_{\mathrm{L}} \boldsymbol{V}\right)=0$,

$$
\begin{aligned}
\frac{\partial\left(p(\phi) \rho_{\mathrm{L}} \boldsymbol{V}\right)}{\partial t}= & -\nabla \cdot\left(p(\phi) \rho_{\mathrm{L}} \boldsymbol{V} \boldsymbol{V}\right)+\nabla \cdot\left(\mu_{\mathrm{L}} \nabla p(\phi) \boldsymbol{V}\right) \\
& -\nabla P+\rho_{\mathrm{L}} \boldsymbol{B}-\frac{h \mu_{\mathrm{L}}(1-p(\phi)) \phi(1-\phi)}{\delta^{2}} \boldsymbol{V}
\end{aligned}
$$

where $\rho_{\mathrm{L}}$ is the melt density, $\mu_{\mathrm{L}}$ the viscosity, and $h$ the friction coefficient for the solid/melt interaction in the diffuse interface; $h=2.757$ [14]. Since the Boussinesq approximation is adopted, the linear form of the body force $\boldsymbol{B}$ can be approximated by

$\boldsymbol{B}=-\left(\beta_{C}\left(C-C_{f}\right)+\beta_{T}\left(T-T_{f}\right)\right) \boldsymbol{g}$,

where $\beta_{C}$ and $\beta_{T}$ are the solutal and thermal expansion coefficients, respectively, and $\boldsymbol{g}$ the gravitational acceleration. In this study, the gravitational direction is pointing downward, i.e., $\boldsymbol{g}=-\boldsymbol{e}_{\mathrm{y}}$. The subscript $f$ corresponds to the reference state and is defined at the inlet condition, i.e., at $y=H$. Also, to neglect kinetic effects, we choose the kinetic coefficient $\mu_{\mathrm{A}}$ based on thin-interface analysis [15]:

$\frac{1}{\mu_{k}}=\eta\left[5 a_{2} / 4\right]\left[\delta / D_{\mathrm{L}}\right]\left[\left(D_{\mathrm{L}} L_{\mathrm{A}} / \alpha C p\right)+|m|(1-k) C\right]$,

where $a_{2}=47 / 75$.

The above equations with a given initial condition can then be solved by an adaptive finite volume method [16], where the minimum grid size $(\Delta x)$ is smaller than the interface thickness $\delta ; \delta=0.25-1 \mu \mathrm{m}$ is used in this study. In this study, a linear temperature profile having a gradient $G$ is assumed. Therefore, the thermal equation, Eq. (2), is not solved.

Before the results and discussion, the phase field simulation without flow is first validated through the comparison with the one by Echebarria et al. [15] for the directional solidification of a SCN alloy; all the physical and simulation parameters can be found in Ref. [15] as well. The calculated steady-state cell shapes at two different interface thicknesses, and the comparison with those obtained by Echebarria et al. [15] are shown in Fig. 3a. The morphology predicted by the well-known SaffmanTaylor shape [17] is also included for comparison. As shown, good agreement is found; the Saffaman-Taylor shape is more accurate near the tip. Further convergence tests on the steady-state tip concentration and tip radius as a function of the interface thickness are shown in Figs. 3b and c, respectively. In Fig. 3b, the solidus concentration is along the cell center; the effective equilibrium coefficient $k_{\text {eff }}$ is also shown. In Fig. 3c, the tip radii are determined by 

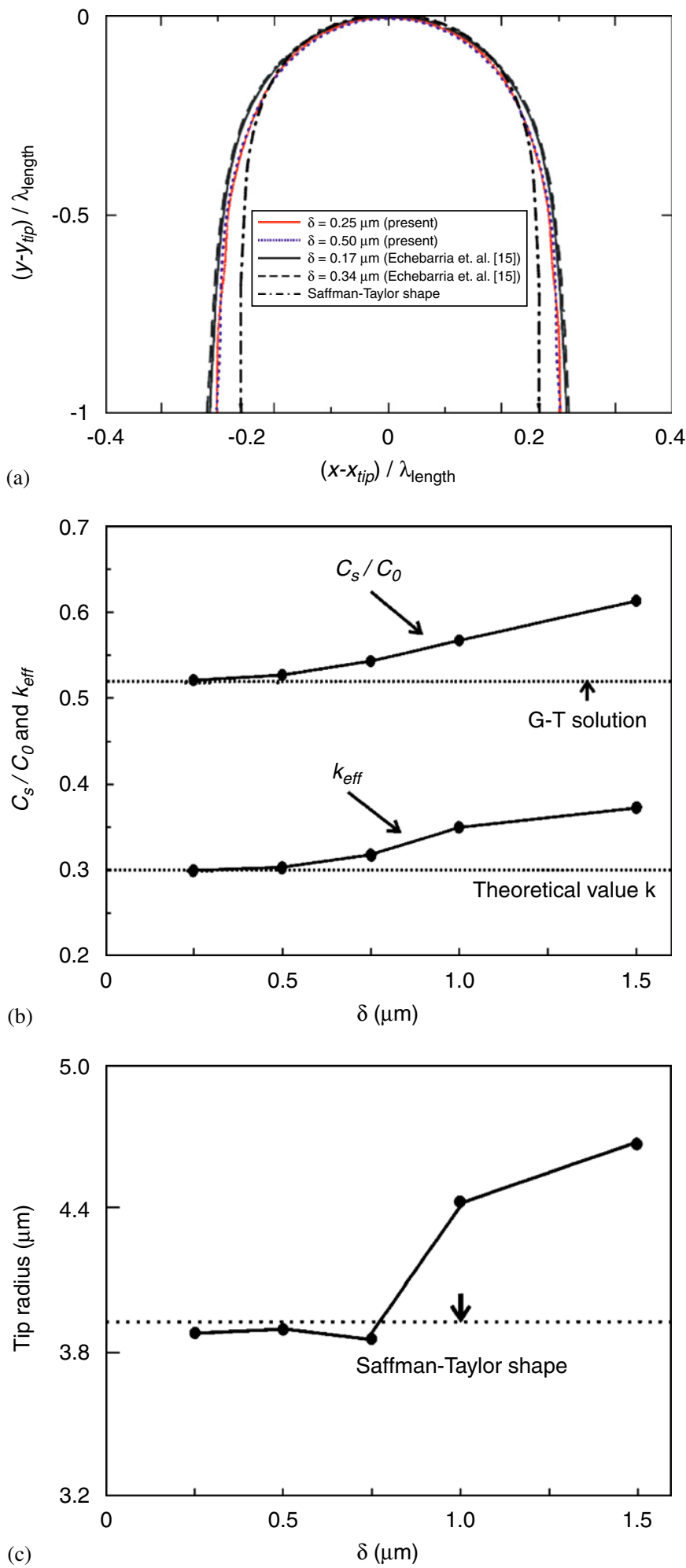

Fig. 3. Comparison of the simulated results for the alloy directional solidification investigated in Ref. [13]: (a) cell shape; (b) the convergence behavior of calculated tip concentration and the effective segregation coefficient with the interface thickness; and (c) the convergence behavior of the calculated tip radii with the interface thickness.

using fourth-order polynomial fitting within $1 \mu \mathrm{m}$ domain around the cell tip. Again, these properties converge nicely as $\delta$ is reduced to $0.5 \mu \mathrm{m}$. The results obtained by using the simple interface model [12] are also in good agreement with the results in Fig. 3. However, since Karma's model has been widely used, for comparison purpose we shall report the simulated results based on their formulation.

\section{Results and discussion}

To investigate the effect of convection, we take the directional solidification of SCN containing $0.1 \mathrm{~mol} \%$ acetone as an example $(k=0.1, m=-222 \mathrm{~K} / \mathrm{mol}$ frac.), while the physical properties are taken from SCN directly. The parameters used in simulation are summarized in Table 1. A linear temperature profile having a gradient $G$ of $100 \mathrm{~K} / \mathrm{cm}$ is used for simulation. In other words, the temperature is assumed to be linear and fixed, i.e., the frozen temperature approximation. In reality, this assumption is reasonable because thermal diffusivity is much larger than the solutal diffusivity. At this concentration, at steady state, based on the MS theory, the critical pulling velocity $V_{\mathrm{c}}$ and wavelength $\lambda_{\mathrm{c}}$ are about $5.7 \mu \mathrm{m} / \mathrm{s}$ and $160 \mu \mathrm{m}$, respectively. This critical velocity and wavelength have been used to examine our simulation, and the agreement is satisfactory $\left(V_{\mathrm{c}}\right.$ less than $1 \%$ and $\lambda_{\mathrm{c}}$ less than $5 \%$ ). To investigate the effect of convection induced by the gravity, we have chosen the pulling speeds lying around the critical velocity for study.

Without gravity, when the pulling velocity $\left(V_{\mathrm{p}}\right)$ is set at $5 \mu \mathrm{m} / \mathrm{s}$, which is lower than critical one, a planar interface is obtained and the axial concentration profile is in excellent agreement with the one-dimensional analytic solution. The calculated concentration field is shown in Fig. 4a. The straight streamlines are due to the material movement with respect to the fixed frame. Once the gravity is introduced, because the acetone is lighter than SCN $\left(\beta_{\mathrm{C}}>0\right)$, a flow cell is induced (convective instability), as shown in Fig. $4 \mathrm{~b}$. Because of the domain size, the

Table 1

Parameters used in simulation

Physical properties of SCN-acetone alloy [6]

Mass diffusivity (liquid side), $D_{\mathrm{L}}$

Mass diffusivity (solid side), $D_{\mathrm{S}}$

Kinetic viscosity, $v$

Gravity acceleration, $\boldsymbol{g}$

Solutal expansion coefficient, $\beta_{C}$

Thermal expansion coefficient, $\beta_{T}$

Melting temperature of pure melt, $T_{m}{ }^{\mathrm{A}}$

Gibbs-Thompson coefficient, $\Gamma$

Anisotropy, $\varepsilon$

Equilibrium partition coefficient, $k$

Slope, $|m|$

Simulation parameters

Domain size

Initial interface position, $y_{\mathrm{i} 0}$

Temperature gradient, $G$

Pulling velocity, $V_{\mathrm{p}}$

Reference length, $l$

Bulk concentration, $C_{0}$

$10^{-9}\left(\mathrm{~m}^{2} / \mathrm{s}\right)$

$10^{-14}\left(\mathrm{~m}^{2} / \mathrm{s}\right)$

$10^{-6}\left(\mathrm{~m}^{2} / \mathrm{s}\right)$

$10\left(\mathrm{~m} / \mathrm{s}^{2}\right)$

$1.8 \times 10^{-3}\left(\mathrm{~mol} \mathrm{\%}{ }^{-1}\right)$

$8 \times 10^{-4}(1 / \mathrm{K})$

$331.24(\mathrm{~K})$

$6.48 \times 10^{-8}(\mathrm{~K} / \mathrm{m})$

0.007

0.1

$2.22(\mathrm{~K} / \mathrm{mol} \%)$

$500 \times 2500\left(\mu \mathrm{m}^{2}\right)$

$500(\mu \mathrm{m})$

$10000(\mathrm{~K} / \mathrm{m})$

$5.0,6.5,12(\mu \mathrm{m} / \mathrm{s})$

$10^{-6}(\mathrm{~m})$

$0.10(\mathrm{~mol} \mathrm{\%})$ 

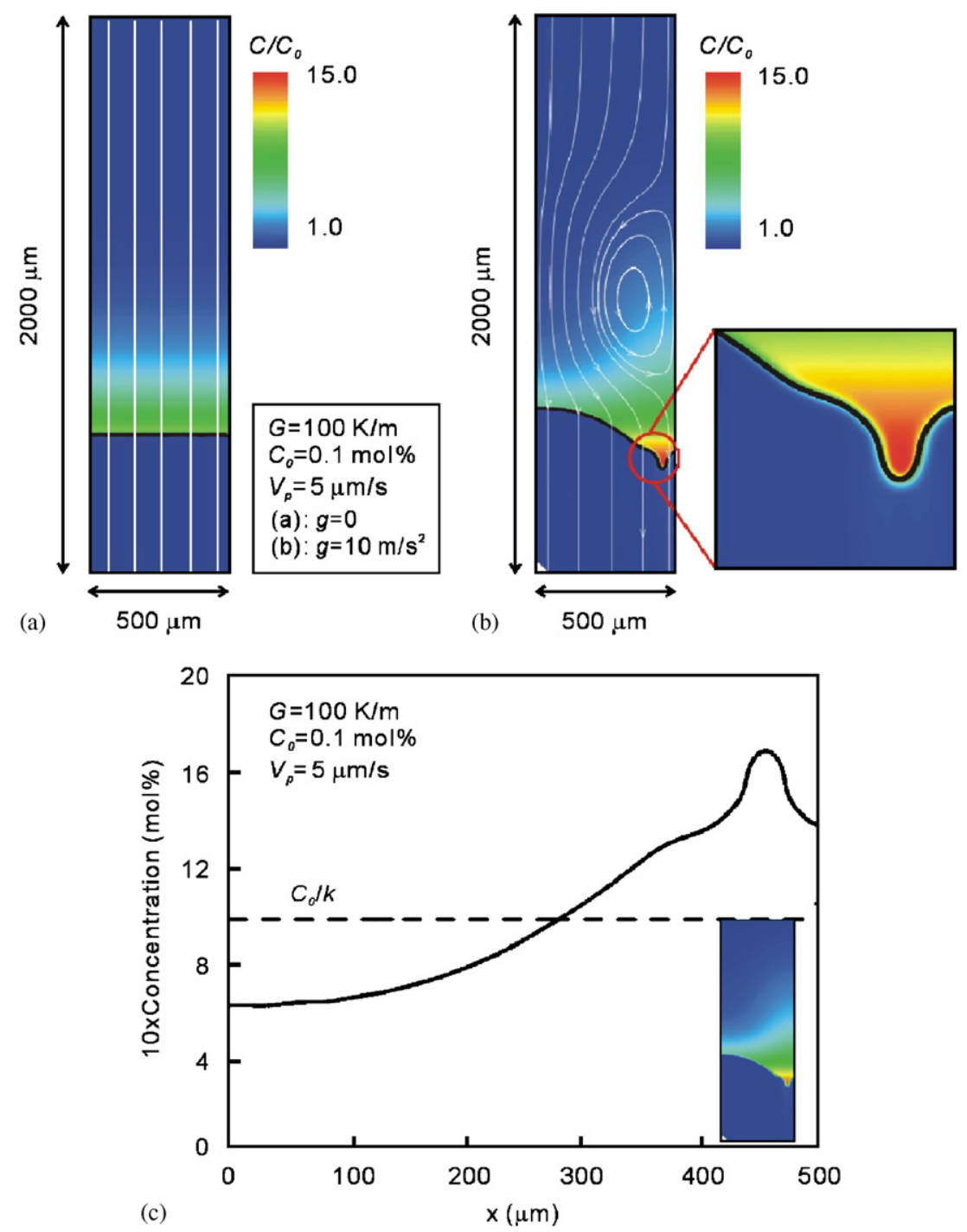

Fig. 4. Effect of gravity for $V_{\mathrm{p}}=5 \mu \mathrm{m} / \mathrm{s}\left(<V_{\mathrm{c}}\right)$ on the flow and concentration fields and the interface shape: (a) without gravity; (b) with gravity; (c) the concentration distributions along the $x$-axis obtained from (a) and (b) at the interface.

wavelength of the flow is two times of the domain width, i.e., $1000 \mu \mathrm{m}$. The lateral acetone segregation induced by the flow also leads to the large deformation of the interface; again, the wavelength of the deformation is $1000 \mu \mathrm{m}$. Interestingly, if we take a close-up look of the interface near the edge in Fig. 4b, a shallow cell (morphological instability) is observed. In other words, the convection due to the gravity, not only causes the long-wavelength deformation (convective mode) as a result of the convective instability, but also the short-wavelength one (morphological mode) due to the morphological instability. The later is caused by the lateral acetone segregation. The higher acetone concentration makes the morphological instability happen earlier, even though the pulling speed is less than the critical one based on the MS theory. The liquid concentration profile at the interface $(\phi=0.5)$ along the $x$-axis is further plotted in Fig. 4c. It is clear that the lateral concentration gradients are caused by the convection. The dashed-line indicating $C_{0} / k$ is the average concentration, which is also the same as the one without gravity because a steady state is reached. In addition, the cell formation also results in a local acetone accumulation. Furthermore, the local interface concentration near the morphological instability seen in Fig. $4 \mathrm{c}$ is in good agreement with the constitutional supercooling criterion [18],

$\left[\frac{C_{0}}{k}\right] V_{\mathrm{p}}>\frac{G D_{\mathrm{L}}}{|m|(1-k)}$.

In Fig. $4 \mathrm{c}$, our $C_{\mathrm{L}} V_{\mathrm{p}}$ for obtaining the morphological instability is found to be in good agreement with the above equation. In fact, the stability function [19] due to the interfacial energy for SCN is near unity. Hence, Eq. (8) gives a good criterion for the morphological breakdown.

If the pulling speed is increased to $6.5 \mu \mathrm{m} / \mathrm{s}\left(>V_{\mathrm{c}}\right)$, the calculated steady-state interface is no longer planar even without gravity. A shallow-cell structure appears as shown in Fig. 5a in the absence of gravity. The wavelength of the shallow cells is about $2 \lambda_{\mathrm{c}} / 3$, i.e., about $100 \mu \mathrm{m}$, which is 

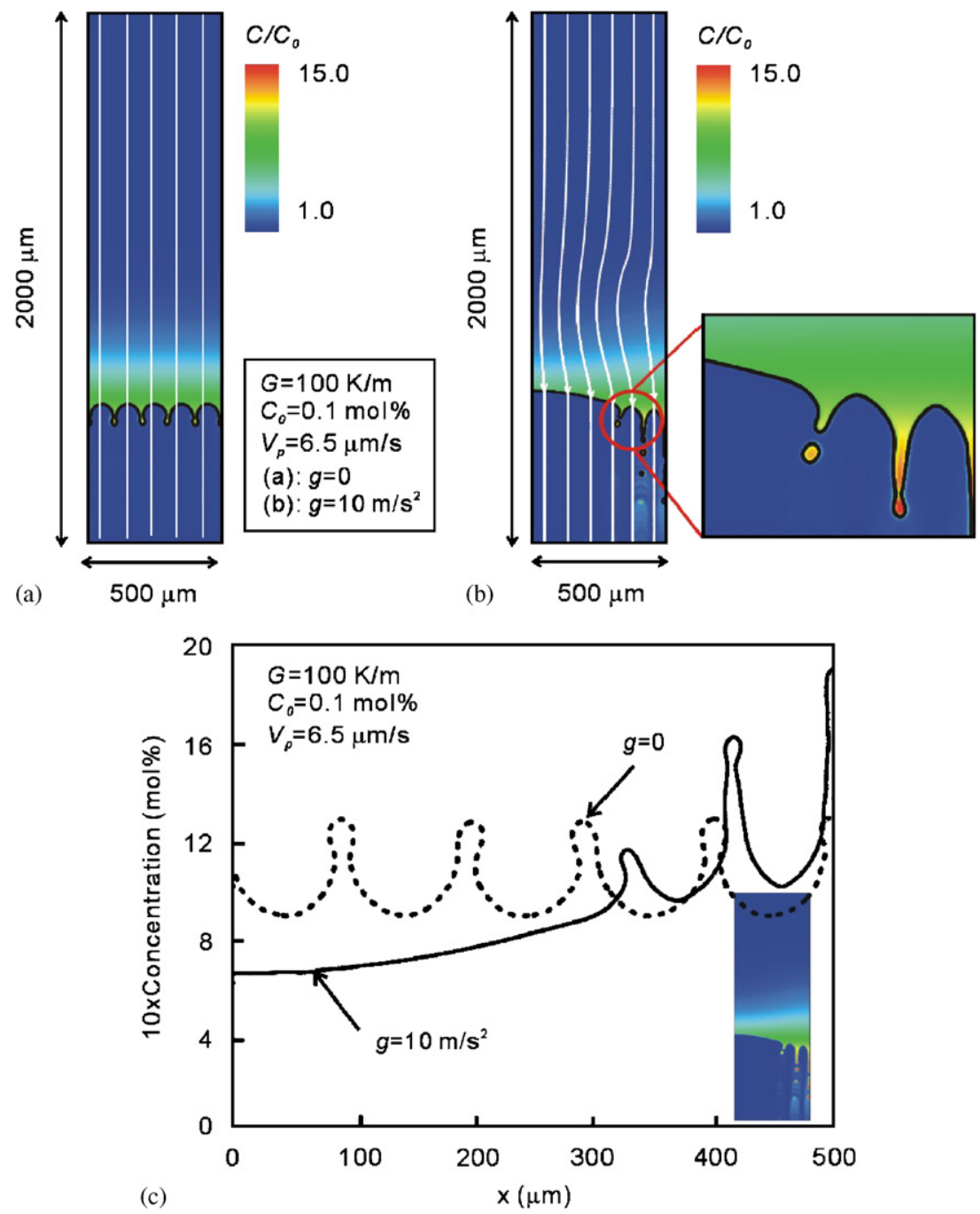

Fig. 5. Effect of gravity for $V_{\mathrm{p}}=6.5 \mu \mathrm{m} / \mathrm{s}$ ( $>V_{\mathrm{c}}$ ) on the flow and concentration fields and the interface shape: (a) without gravity; (b) with gravity; (c) the concentration distributions along the $x$-axis obtained from (a) and (b) at the interface.

consistent with a bifurcated branch near the onset in Ref. [20]. With buoyancy convection, the streamlines are slightly distorted; if the pulling is subtracted from the streamline values, a counterclockwise flow cell appears. Similar to the one in Fig. 4b, the flow induces the lateral concentration segregation. As a result, the shallow cells disappear on the left of the domain due to the reduced acetone concentration. Meanwhile, two cells remain and become much deeper in the right corner, again as a result of higher acetone concentration there. Some pitch-off solute droplets are also observed in the bottom of the cell glooves. As a result of the physical solute trapping, the lateral solute gradients for the buoyancy flow are slightly reduced. And this could be a reason that the overall flow intensity in the present case seems to be smaller than that in Fig. 4. On the other hand, due to the straightened effect of the higher pulling speed, the flow cell is not obvious.

Fig. $5 \mathrm{c}$ presents the acetone concentration obtained from Figs. $5 \mathrm{a}$ and $\mathrm{b}$ along the $x$-axis. Because the pulling speed is larger, the critical concentration is smaller indicating that the region having morphological instability is wider, as compared to that in Fig. 4c. Again, the critical $C_{\mathrm{L}} V_{\mathrm{p}}$ for having the morphological instability is found in good agreement with Eq. (8).

Furthermore, if the pulling speed is further increased, the solutal boundary layer is thinner, on the order of $D_{\mathrm{L}} / V_{\mathrm{p}}$, and the convection in the lateral direction could be suppressed due to the smaller destabilized regime, as shown in Fig. 6 at $V_{\mathrm{p}}=12 \mu \mathrm{m} / \mathrm{s}$. The destabilizing driving force, the Rayleigh number, is proportional to $\left(D_{\mathrm{L}} / V_{\mathrm{p}}\right)^{3}$ for solutal convection. Therefore, as the pulling speed increases, the convective speed due to the gravity decreases. Beside the increase in the cell depth, the effect of gravity becomes insignificant. The wavelength is about $77 \mu \mathrm{m}$, which is about $\lambda_{\mathrm{c}} / 2$. In the present simulation, the buoyant melt speed is on the order of the crystal growth speed (pulling speed).

Finally, as we plot the MS boundary in Fig. 7, the physical feature of the previous simulations becomes clear. Since the length in the $x$-direction is fixed at $500 \mu \mathrm{m}$, the 


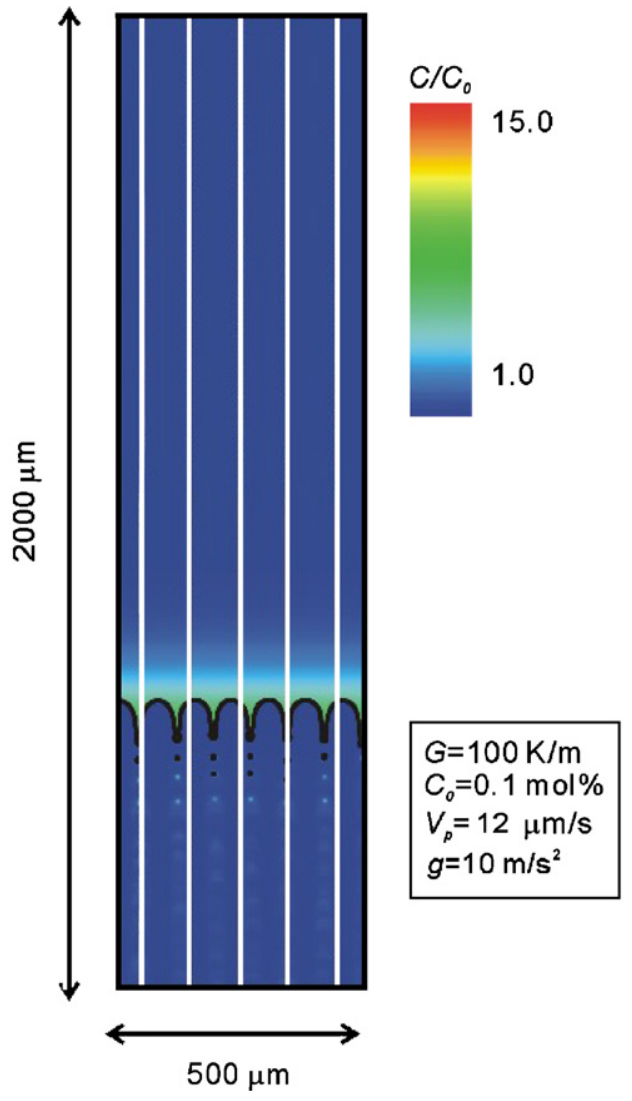

Fig. 6. Calculated flow and solute fields, as well as the interface shape, for $V_{\mathrm{p}}=12 \mu \mathrm{m} / \mathrm{s}\left(\gg V_{\mathrm{c}}\right)$.

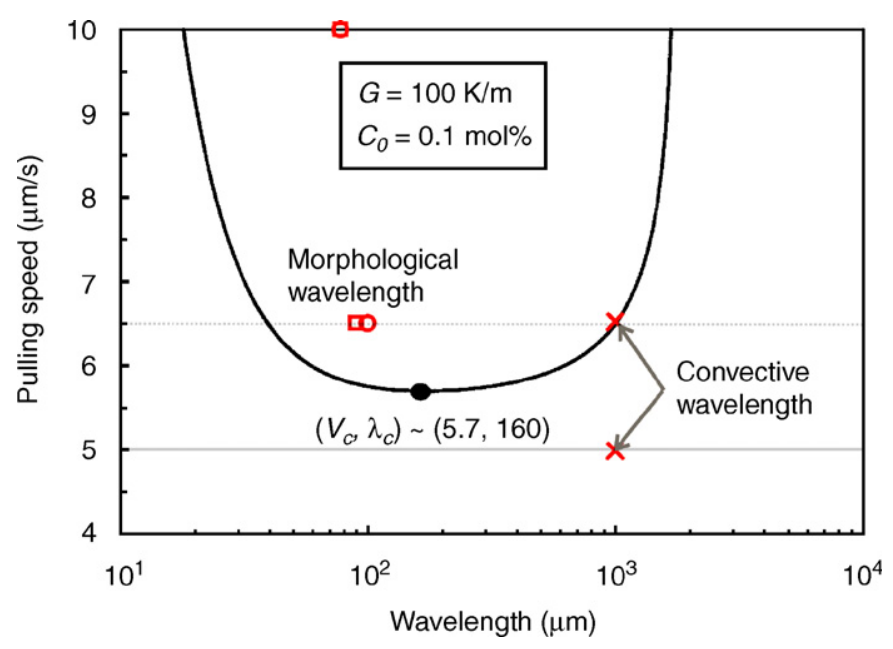

Fig. 7. Calculated wavelengths and the comparison with the MS boundary; the cross symbols indicate the convective mode, circle symbols the morphological mode, and the square symbols the morphological mode under buoyancy convection.

simulated flow cell has a wavelength of $1000 \mu \mathrm{m}$, and the induced interface deformation has the same wavelength as well. This wavelength caused by the buoyancy effect is much longer than that caused by the constitutional supercooling (about $\lambda_{\mathrm{c}} / 2$ ). In other words, the coexistence of the long- and short-wavelength modes is found in the present investigation, which is not seen from the previous linear stability prediction and the non-linear finite element simulations.

\section{Conclusions}

We have successfully used the phase field model to simulate the thermal-solutal effect on the interface morphology during directional solidification of an SCN/ acetone alloy. By using the phase field simulation, the deformation of the interface and the species/momentum transports can be treated easily. In the presence of gravity, the solidification interface can become unstable even at a stable regime predicted by Mullins-Sekerka theory due to the lateral solutal segregation induced by the hydrodynamic instability. The shallow cells near the onset of the instability are affected by the flows as well. The cells with different wavelengths and depths can coexist due to the flow-induced segregation. The coupling and co-existence of the long- (convective) and short- (morphological) wavelength modes are found and are illustrated for the first time by the phase field simulation.

\section{Acknowledgment}

This research is sponsored by the National Science Council of Taiwan.

\section{References}

[1] W.W. Mullins, R.F. Sekerka, J. Appl. Phys. 35 (1964) 444.

[2] S.R. Coriell, M.R. Cordes, W.J. Boettinger, R.F. Sekerka, J. Crystal Growth 49 (1980) 13.

[3] S.R. Coriell, G.B. McFadden, J. Crystal Growth 94 (1989) 513.

[4] R.J. Schaefer, S.R. Coriell, Metall. Trans. A 15 (1984) 2109.

[5] C.W. Lan, C.Y. Tu, J. Crystal Growth 220 (2000) 619.

[6] M. R. Mehrabi, Ph.D. Thesis, Massachusetts Institute of Technology, 1994.

[7] J.A. Warren, C. Beckermann, A. Karma, Annu. Rev. Mater. Res. 32 (2002) 163.

[8] A. Karma, W.J. Rappel, Phys. Rev. E 53 (1996) 3017.

[9] C.W. Lan, Y.C. Chang, J. Crystal Growth 250 (2003) 525.

[10] N.A. Ahmad, A.A. Wheeler, W.J. Boettinger, G.B. McFadden, Phys. Rev. E 58 (1998) 3436.

[11] A. Karma, Phys. Rev. Lett. 87 (2001) 115701.

[12] C.J. Shih, M.H. Lee, C.W. Lan, J. Crystal Growth 282 (2005) 515.

[13] C.W. Lan, C.J. Shih, M.H. Lee, Acta Mater. 53 (8) (2005) 2285.

[14] C. Beckermann, H.-J. Diepers, I. Steinbach, A. Karma, X. Tong, J. Comput. Phys. 154 (1999) 468.

[15] B. Echebarria, R. Folch, A. Karma, M. Plapp, Phys. Rev. E 70 (2004) 061604.

[16] C.W. Lan, C.C. Liu, C.M. Hsu, J. Comput. Phys. 178 (2002) 464.

[17] P.G. Saffman, G.I. Taylor, Proc. Roy. Soc. London A 254 (1958) 312.

[18] W.A. Tiller, K.A. Jackson, J.W. Rutter, B. Chalmers, Acta Matall. 1 (1953) 428.

[19] R.F. Sekerka, in: W. Bardsley, D.T.J. Hurle, J.B. Mullin (Eds.), Crystal Growth: An Introduction, Elsevier, North Holland (Chapter 15), 1973.

[20] K. Tsiveriotis, R.A. Brown, Phys. Rev. B 48 (1993) 13495. 